\title{
Transposition
}

Musique et Sciences Sociales

$6 \mid 2016$

Lignes d'écoute, écoute en ligne

\section{Proust au téléphone}

Placer la Recherche sur écoute

Jesse Dylan McCarthy

\section{OpenEdition}

1 Journals

\section{Édition électronique}

URL : http://journals.openedition.org/transposition/1491

DOI : 10.4000/transposition. 1491

ISSN : 2110-6134

Éditeur

CRAL - Centre de recherche sur les arts et le langage

Référence électronique

Jesse Dylan McCarthy, «Proust au téléphone », Transposition [En ligne], 6 | 2016, mis en ligne le 20 mars 2017, consulté le 31 juillet 2019. URL : http://journals.openedition.org/transposition/1491 ; DOI : 10.4000/transposition. 1491

Ce document a été généré automatiquement le 31 juillet 2019

\section{cc) () (?}

La revue Transposition est mise à disposition selon les termes de la Licence Creative Commons Attribution - Partage dans les Mêmes Conditions 4.0 International. 


\title{
Proust au téléphone
}

\author{
Placer la Recherche sur écoute
}

Jesse Dylan McCarthy

"Les vrais livres doivent être les enfants non du
grand jour et de la causerie mais de l'obscurité et
du silence. "
(Le Temps retrouvé, III, p. 898)

1 Dans la chambre tapissée de liège, l'œuvre prend forme : les mots s'enchaînent, la syntaxe s'enchâsse, les «paperolles » se convertissent en mille-feuilles. Le fond de cette écriture est une nuit insonorisée, un silence insolite au cœur même de la capitale où Proust s'est renfermé comme dans un bocal hermétiquement scellé. Pendant de longues heures, les seuls bruits sont le faible grattement de la plume et la toux irrégulière qui marque les pauses. Et pourtant, dans les pages de la Recherche, déferle toute la musique du monde, d'une époque : tout le bourdonnement de la vie quotidienne, le bavardage de soirées interminables, le pullulement de voix familières et grossières, le rire des jeunes filles au bord de la mer, le cri des vendeurs de journaux; tout un fracas qui s'élève et résonne autour de nous comme dans le grand hall des départs de la gare de Lyon.

2 Comment l'écrivain, cet artisan dévoué du silence, reconstitue-t-il alors le monde sensible? Celui de l'atmosphère variable du temps terrestre, de ces empreintes uniques laissées par la voix humaine, de la rumeur des tramways et de la ville, de la jacasserie mondaine? La question est loin d'être purement déontologique. Le texte proustien est sans cesse en train de nous immerger dans sa passoire auditive, d'imaginer une écoute active, de placer pour ainsi dire ses protagonistes sur écoute - et même en situation de surécoute, pour employer ce néologisme proposé par Peter Szendy qui désignerait « une intensification de l'écoute, comme sa forme hyperbolique, portée à incandescence, à sa pointe la plus extrême et la plus active ${ }^{1}$ ».

3 Sur l'importance de la musique dans la Recherche, de Wagner, de la « petite phrase » de la sonate de Vinteuil, beaucoup d'encre a coulé. Mais l'obsession aurale, l'imaginaire de l'écoute en tant que tel, pourtant très évident, n'ont pas été aussi souvent remarqués. Il est étonnant, par exemple, qu'un livre comme celui de Jean-Pierre Richard, étude essentiellement phénoménologique de la poétique proustienne, ne comporte aucun 
chapitre sur les sons, le timbre, les rapports auditifs's. Il n'empêche que c'est toute une poésie de l'aural qui se diffuse à travers l'œuvre et que les traces de cette chambre de résonances sont lisibles.

4 Une lecture à l'écoute de la Recherche découvre rapidement des faisceaux qui transpercent les frontières entre la vérité de l'auteur et la fiction de ses personnages. Le « silence » des «vrais livres» qu'invoque le narrateur du Temps retrouvé, par exemple, ne peut être lu purement à titre métaphorique; sa prescription non plus, qui peine à démentir une fascination pour la causerie mondaine. Pour démêler les modes d'écoute, il faut alors distinguer deux niveaux de relais entre sources et récepteurs.

Premièrement, il y a le silence de la genèse de l'œuvre, c'est-à-dire celui de l'auteur à l'écoute de son propre texte. Le cloisonnement de la chambre du 102, boulevard Haussmann, avec sa fameuse insonorisation en plaques de liège, imposa un silence, et donc un rapport à l'écoute, qui fut des plus intimes. C'est Céleste Albaret qui en témoigne dans ses mémoires ${ }^{3}$. Placer la Recherche sur écoute, c'est d'abord garder à l'esprit ce silence de composition dont les traces se manifestent dans l'enregistrement affiné et presque pinailleur des arrière-plans proustiens, comme dans l'ouverture célèbre de La Prisonnière, où les changements de saison et les plus infimes rumeurs de la rue sont perçus avec une finesse sismographique par le narrateur à son réveil ${ }^{4}$.

6 Cet effet de stéthoscope, d'écoute médiatisée à partir d'une chambre et à travers une paroi, que ce soit l'espionnage d'Albertine à Paris ou les mouvements de sa grand-mère à Balbec, nous le retrouvons régulièrement tout au long du roman. En suivant une autre suggestion de Peter Szendy ${ }^{5}$, je propose d'appeler ces écoutes des instances "d'auscultation médiate », selon la terminologie de Laennec, médecin et inventeur du stéthoscope, qui emploie la formule pour la différencier d'une auscultation immédiate, où le médecin place directement son oreille sur le corps du malade. L'intérêt de cette méthode, décrite dans son traité De l'auscultation médiate (1819), c'est de transformer la perception auditive en un savoir quasi visuel grâce à l'amélioration de son pouvoir de distinction diagnostique ${ }^{6}$. Laennec est d'ailleurs fasciné par la précision obtenue à l'écoute grâce à son instrument et ses descriptions des crépitements de bulles dans les poumons de ses patients tournent plus d'une fois au littéraire. L'auscultation, qui consiste à écouter attentivement à travers une paroi, est donc aussi médiate dans la mesure où l'acte de vérification modifie le contenu ${ }^{7}$. C'est la naissance d'une perception indirecte et, par conséquent, imaginative.

7 Deuxièmement, on peut distinguer l'écoute des personnages de la Recherche entre eux : ce sont alors des écoutes assujetties à diverses mésententes, déviations et brouillages. Pour comprendre les enjeux de cette dimension de l'écoute au sein du roman, il n'y a pas de meilleure cible que les échanges téléphoniques, ainsi que toute la réflexion proustienne autour de cet appareil qui bouleverse les communications de la Belle Époque, au sein d'un monde désormais relié à distance par une nouvelle médiation surgissant au milieu des habitudes d'un siècle révolu.

8 Afin d'examiner de près l'une des scènes d'auscultation médiate les plus intéressantes du roman, on se penchera d'abord sur l'arrivée du narrateur à Doncières, scène étrange mais révélatrice qui se déroule vers le début du Côté de Guermantes. Nous nous tournerons ensuite vers deux autres scènes téléphoniques cruciales dans la Recherche: celle de l'appel manqué avec la grand-mère, également à Doncières ; et, enfin, la scène téléphonique avec Albertine, qui suit la soirée de la princesse de Guermantes dans Sodome et Gomorrhe, une scène dont la finesse et l'originalité ont rarement été appréciées. 


\section{La chasteté du silence}

Au début du Côté de Guermantes, nous retrouvons le narrateur en visite chez son ami SaintLoup à la garnison de Doncières. Sur place, la rencontre entre les deux amis est interrompue avant même qu'elle n'ait commencé :

Il faut que je dise un mot au capitaine, me chuchota Saint-Loup ; soyez assez gentil pour aller m'attendre dans ma chambre, c'est la seconde à droite, au troisième étage, je vous rejoins dans un moment ${ }^{8}$.

Proust suspend le récit et laisse son narrateur seul encore un moment dans ce nouveau décor inconnu. D'un point de vue narratif, ce délai serait habituellement utilisé pour permettre au narrateur de décrire au lecteur la chambre de Saint-Loup, développant ainsi sa caractérisation avant que les deux personnages ne s'engagent dans une conversation qui va faire avancer l'intrigue. On s'attendrait donc à découvrir ici une description plus ou moins générique d'une chambre avec ses attributs bourgeois, indices de rang et de goût, bref, une chambre qui nous aide à mieux cerner et situer un personnage, à la manière de Balzac ou de Stendhal. Mais au lieu d'entrer directement dans la chambre, le narrateur reste figé à la porte, troublé par ce qu'il entend de l'autre côté :

Je restai un instant devant la porte fermée, car j'entendais remuer ; on bougeait une chose, on en laissait tomber une autre ; je sentais que la chambre n'était pas vide et qu'il y avait quelqu'un. Mais ce n'était que le feu allumé qui brulait. Il ne pouvait pas se tenir tranquille, il déplaçait les buches et fort maladroitement ${ }^{9}$.

11 L'oreille tente de fixer le bourdonnement masqué et feutré par la porte ; mais, même quand il vérifie son origine dans le feu, le son ne peut être fixé. Le bruit se déplace, frétillant, suggérant une présence humaine, comme si c'était le résidu spectral de SaintLoup lui-même, dont le personnage est aussi voltigeant que son monocle. Ce spectre aural se voit confirmé par métonymie quand l'écoute est transférée du feu à un autre objet cher à Saint-Loup, sa montre :

J'entendais le tic tac de la montre de Saint-Loup, laquelle ne devait pas être bien loin de moi. Ce tic tac changeait de place à tout moment car je ne voyais pas la montre; il me semblait venir de derrière moi, de devant, d'à droite, d'à gauche, parfois s'éteindre comme s'il était très loin. Tout d'un coup je découvris la montre sur la table. Alors j'entendis le tic tac en un lieu fixe d'où il ne bougea plus. Je croyais l'entendre à cet endroit là ; je ne l'y entendais pas, je l'y voyais, les sons n'ont pas de lieu ${ }^{10}$.

Dans cette progression confuse, l'écoute du narrateur est inversée par rapport au scénario précédent. Maintenant, il est dans la chambre et il croit entendre un bruit au dehors. Son auscultation devient presque animale. Il balaye la chambre de Saint-Loup comme la chauve-souris chassant dans sa caverne grâce à sa faculté dite d'« écholocation ", tentant de cerner la source, de fixer son emplacement dans l'espace. Mais l'acte même d'auscultation conduit à la désorientation sensorielle; l'auditif a été découplé de sa source et c'est seulement quand l'écoute imaginaire a été falsifiée que le narrateur découvre le fait que la montre a simplement été posée sur la table. «Les sons n'ont pas de lieu. » Le narrateur insiste non sur l'erreur de sa perception, mais sur son paradoxe. Si l'auscultation médiate l'a conduit à une erreur perceptive, elle révèle en revanche une vérité psychologique: les données de sa perception ne sont pas objectifs, mais déformées par l'intérêt subjectif, dont la cible n'a évidemment jamais été la montre, mais l'homme, l'ami insaisissable. 
13 On pourrait encore à ce stade imaginer Proust décrire la chambre d'une manière conventionnelle. Au lieu de cela le texte nous provoque par le détour d'une hypothèse fantasque. Il propose d'imaginer le monde du silence qu'habite un malade « auquel on a hermétiquement bouché les oreilles ${ }^{11} »$. La chambre de Saint-Loup n'est qu'un prétexte pour une digression sur les possibilités créatives de l'écoute pure. Le récit à la dérive est embarqué dans un monde entièrement auditif. Proust se met lentement à baisser le volume :

Alors, que le malade lise, et les pages se tourneront silencieusement comme si elles étaient feuilletées par un dieu. La lourde rumeur d'un bain qu'on prépare s'atténue, s'allège et s'éloigne comme un gazouillement céleste. Le recul du bruit, son amincissement, lui ôtent toute puissance agressive à notre égard; affolés tout à l'heure par des coups de marteau qui semblaient ébranler le plafond sur notre tête, nous nous plaisons maintenant à les recueillir, légers, caressants, lointains comme un murmure de feuillages jouant sur la route avec le zéphyr ${ }^{12}$.

14 Cette « lourde rumeur » et le "gazouillement céleste » tissent directement dans le texte le timbre du son d'un bain coulé dans la chambre d'à côté. Mais surtout, ce "gazouillement céleste", expression assez recherchée, n'a rien d'un accident. Le narrateur insistera dans Le Temps retrouvé sur le fait que ce mot magnifique apparait dans sa phrase préférée des Mémoires d'Outre-Tombe de Chateaubriand ${ }^{13}$. Il nous faut donc rester attentivement à l'écoute des intertextes de la Recherche ainsi que du jeu autobiographique. On ne peut douter que Proust joue ici aussi, dans un esprit de calembour, sur le nom de sa bonne Céleste Albaret, qui fut manifestement celle qui préparait les bains de l'auteur.

15 D'ailleurs cette scène (hypothèse toujours continuée, parenthèse encore dilatée) démontre à quel point l'auscultation est une figure qui se rapporte à la genèse de l'œuvre. Car qui est ce malade supposé sinon l'auteur maladif? Pour lui, chaque vibration, chaque sonnerie est précieuse. La prose s'allège comme sa fantaisie et il ne peut supprimer un réflexe ludique, son côté comique et enfantin.

Le malade passe aux jeux et l'on ne peut s'empêcher d'imaginer Céleste en train de monter le thé et de trouver Marcel jouant avec ses tampons, faisant des oreilles de chien, les alternant pour mieux savourer l'effet stéréoscopique: «et en augmentant, en relâchant les tampons d'ouate, c'est comme si on faisait jouer alternativement l'une et l'autre des deux pédales qu'on a ajoutées à la sonorité du monde extérieur ${ }^{14}$ ». Si jusqu'ici le texte fait référence à des bruits (le tramway, un piano) qui sont plausibles dans l'environnement de Doncières, quand arrive l'image savourée et minutieusement détaillée d'un malade tellement absorbé dans sa rêverie qu'il laisse déborder son lait en ébullition, l'on ne peut plus prétendre à la métaphore diégétique; et l'auteur semble le reconnaitre avec un petit clin d'œil au lecteur dans lequel il nous laisse surprendre aussi la voix de Céleste en train de le gronder :

émergeant à peine d'une mer blanche après ce mascaret lacté, il serait obligé d'appeler au secours sa vieille bonne qui, fût-il lui-même un homme politique illustre ou un grand écrivain, lui dirait qu'il n'a pas plus de raison qu'un enfant de cinq ans ${ }^{15}$.

17 Écouter permet donc de passer d'une pièce à l'autre au sein de la fiction, mais aussi de transcender l'espace fictif lui-même ; de passer d'une chambre à Doncières à une chambre boulevard Haussmann, et vice-versa.

Ces tampons d'ouate rappellent aussi l'étrange simultanéité d'écoute que permet le théâtrophone, inventé par Clément Ader en 1881 et diffusé dès 1890 dans tout Paris par la 
Compagnie du Théâtrophone ${ }^{16}$. Celui-ci relie les principaux théâtres - le théâtre Français, l'Opéra Garnier et l'Opéra Comique - à un bureau central situé 23 , rue Louis le Grand, qui retransmet les spectacles à ses abonnés à travers le réseau téléphonique parisien. Proust obtient un abonnement début 1911 et, malgré une qualité sonore apparemment souvent médiocre, il s'en éprend avec un enthousiasme ardent ${ }^{17}$. En effet, ce dispositif ingénieux multiplie et médiatise les points d'écoute. Il permet à son abonné de placer un spectacle public sur écoute privée, confondant ainsi les sources sonores par un désemboîtement qui dissout la matérialité de l'espace tout en préservant l'invisibilité, le voyeurisme de l'espion. C'est un dispositif qui reproduit un spectacle médiatisé dans les conditions de la «surécoute ", à l'instar de la sonorité délocalisée évoquée dans le passage de la chambre de Saint-Loup.

Le texte proustien manifeste non seulement une jouissance particulière de la "surécoute ", mais il en fait un fétiche, poursuivant les dislocations et surimpressions sonores avec une soif répétitive et auto-érotique. En résulte une auscultation qui se déploie sur un plan infiniment extensible, qui cherche son aboutissement dans l'extrême, dans l'expérience limite, c'est-à-dire le monde du sourd, le monde du silence absolu. La chambre de Saint-Loup, auscultée avec délice pour échafauder une rêverie d'opéracomique, s'épand finalement en un paradis artificiel :

c'est avec délices qu'il se promène maintenant sur une Terre presque édénique où le son n'a pas encore été créé. Les plus hautes cascades déroulent pour ses yeux seuls leur nappe de cristal, plus calmes que la mer immobile, pures comme des cataractes du Paradis. Comme le bruit était pour lui, avant sa surdité, la forme perceptible que revêtait la cause d'un mouvement, les objets remués sans bruit semble l'être sans cause [...]. D'eux mêmes ils s'envolent comme les monstres ailés de la préhistoire ${ }^{18}$.

Pourquoi ce fantasme de l'espace intérieur, cette rêverie bizarre de l'assourdi, du degré zéro de l'écoute? Deleuze avait déjà remarqué à quel point la Recherche est hantée par le primitif, par un retour à des états vierges : « Le monde enveloppé de l'essence est toujours un commencement du Monde en général, un commencement de l'univers, un commencement radical absolu ${ }^{19}$. "Certes, mais la scène édénique n'est pas seulement liée ici à une genèse abstraite : elle l'est aussi, directement, à un foisonnement de l'imaginaire chez un patient pour qui la "chasteté du silence» imposé par la maladie facilite un renversement auscultatoire, "un virage par lequel l'œil devient une oreille ${ }^{20}$ » dont le narrateur proustien tire avantage pour mieux tâtonner, selon une écoute qui privilégie surtout l'épicurisme et le délassement.

21 Ce délai momentané, ce passage de rêverie dans la chambre de Saint-Loup n’a finalement rien de très chaste ; c'est une rêverie érotique soutenue par les nouvelles technologies de médiation, un opéra bouffe brouillant la scène domestique avec des colorations wagnériennes. L'imaginaire foisonnant du narrateur se dépense dans l'auscultation qui se révèle comme un instrument d'invention, mais aussi de réflexion approfondie sur l'érotisme de l'écoute en soi-même, sur l'écriture comme instrument d'auscultation médiate entre un lecteur et un texte, ce paradis silencieux de la lecture.

\section{Les Messagères de la parole}

Le «drame du téléphone » est déjà représenté dans l'Urtext de la Recherche, dans les pages de Jean Santeuil. La scène clef se déroule durant le séjour de Jean à la station balnéaire de 
Beg-Meil. L'origine autobiographique de ce passage, rapporté dans la biographie de George Painter, serait le séjour de Proust à Fontainebleau en compagnie de Lucien Daudet, en octobre 1896. C'est par un jour de mauvais temps et de fâcherie entre les amis qu'un Proust déjà malingre cherche désespérément à avoir sa mère au téléphone ${ }^{21}$. Proust saisit d'emblée l'importance de cet épisode et son intérêt romanesque. L'expérience de la voix désincarnée qu'impose le medium téléphonique offre au jeune écrivain un défi technique mais aussi des opportunités offertes par son usage drôlement imparfait, gouverné par le malentendu, les contretemps, les ( $\mathrm{r}$ )accrochages.

Aux frontières de cet espace médiatisé où l'étiquette n'a pas encore pris ses repères, le mélodrame et le pathos sont continuellement en proie au comique et côtoient la farce. Dans Jean Santeuil, Proust met en scène tous les éléments de base (interruptions, légers contretemps), mais le ton reste dominé par le pathétique, par une note de désespoir tragique :

Alors il se représente sa mère sonnant au téléphone, l'appelant, ne comprenant pas pourquoi Jean ne lui répond pas [...]. Mais commotionnant, clair, voici le timbre qui sonne, résonne, semble courir ça et là. Vite il met le tube à l'oreille. La voix forte et dure d'un garçon: «Est-ce M. Santeuil ?» Sans doute on parle pour sa mère, pendant qu'on lui fait prendre le cornet, qu'elle se hâte toute troublée. Une autre voix forte et dure d'un autre garçon. Puis tout d'un coup - c'est comme si tout le monde s'étant allé de la chambre il tombait dans les bras de sa mère - vient là tout contre lui, si douce, si fragile, si délicate, si claire, si fondue - un petit morceau de glace brisée - la voix de sa mère. «C'est toi, mon chéri ? " C'est comme si elle lui parlait pour la première fois, comme s'il la retrouvait après la mort dans le paradis. Car pour la première fois, il entend la voix de sa mère ${ }^{22}$.

Proust étire l'incident pour en extraire toute la moelle dramatique. La voix isolée dans l'espace-temps téléphonique ne se résume pas à la transmission de données; elle établit une présence dialogique qui confirme l'écouteur autant que l'écouté. Si le temps et l'espace s'évanouissent sous la parole reliée à distance, paradoxalement la même formule assure le contrecoup d'une sensation de séparation d'autant plus aiguë. Cette angoisse de la séparation est d'ailleurs toujours reliée à une présence féminine qui est recherchée à l'appareil : la voix maternelle, douce et fondue, la parole aimantée des standardistes.

Pour Santeuil, raccrocher représente un drame œdipien. Chez le narrateur de la Recherche, en revanche, l'appel téléphonique est traité d'une manière plus complexe, avec un plus large ensemble de supplices, étrangement entremêlés de loisirs. Il y décèle, pour la première fois, le merveilleux, «l'admirable féerie à laquelle quelques instants suffisent pour qu'apparaisse près de nous, invisible mais présent, l'être auquel nous voulions parler ${ }^{23}$ ». Cet intérêt pour le merveilleux qui se cache dans le quotidien préfigure celui des surréalistes, qui feront notamment du téléphone, ainsi que du journal, les objets phares d'une modernité confondant les pouvoirs surnaturels avec la banalité d'une vie urbaine remplie de choses et de paroles échangées en masse.

Saisissant son pouvoir quasi talismanique, Proust baptise le téléphone : "la planchette magique». Saurait-on mieux décrire les objets de télécommunication en vogue aujourd'hui ? Pour le narrateur, ce royaume féerique est peuplé par les standardistes (aujourd'hui un peu démodées ou bannies dans de lointains pays en voie de développement) qui lui offrent une comédie piquée d'un brin de sensualité, de téléphone rose : elles sont des "Vierges Vigilantes », des «Danaïdes », d'« Ironiques Furies », « les servantes toujours irritées du Mystère, les ombrageuses prêtresses de l'Invisible, les Demoiselles du téléphone !» Il y a un aspect catin à ces échanges, une tension érotique 
qui rapproche la cabine téléphonique de la maison close. Proust a su remplacer le ton pathétique et larmoyant de Jean Santeuil avec une confiance humoristique et lyrique. Dans la Recherche, il démultiplie les angles de l'expérience et c'est avec une légèreté ironique qu'il aborde tout ce qu'il y a de grave et de drôle aux frontières des nouveaux modes de communication.

Mais le téléphone, c'est aussi une sorte de propylée où retentissent les voix avant d'être enlevées au royaume des morts. C'est un fait accentué par les fragilités de la vieillesse, et qui surgit à l'esprit du narrateur quand il tente de communiquer avec sa grand-mère à l'appareil. La voix de ceux qui nous sont chers, lorsqu'elle retentit sans qu'ils soient à notre portée, est une expérience fantomatique qui les place à mi-chemin, dans une sorte de purgatoire entre la vie et la mort : «Présence réelle que cette voix si proche - dans la séparation effective! Mais anticipation aussi d'une séparation éternelle ${ }^{24}$ !» Proust évoque explicitement Orphée et son cri répété - «Grand-mère, grand-mère!» renforce l'intuition de la mort qui s'approche, le désespoir d'une voix qui tombe dans le néant.

L'auscultation est poussée aux bornes d'une recherche mythique. Proust avait déjà l'intuition que le téléphone est un instrument langagier et que le dévoilement qu'il opère fait non seulement découvrir le grain de la voix, mais le met en scène, produisant un excédent sujet aux décryptages, dans un circuit instable qui tente de cerner une présence toujours en défaut. C'est le jaillissement continu d'une absence au sein même de la présence, d'une fêlure, comme celle de la voix de sa grand-mère : «j'y remarquais pour la première fois les chagrins qui l'avaient fêlée au cours de la vie ${ }^{25}$ \%. Le téléphone découpe la voix, aiguisant non le rapprochement mais la séparation : «cet isolement de la voix était comme un symbole, une évocation, un effet direct d'un autre isolement, celui de ma grand-mère, pour la première fois séparée de $\operatorname{moi}^{26}$ ». Le côté sombre du merveilleux téléphonique, c'est cette violence symbolique introduite par la médiation incorporelle, la séparation des êtres par les instruments qui sont censés les relier.

L'antidote symbolique d'une modernité où la médiation provoque notre isolement, c'est la voix de la locomotive : le cri fougueux des trains qui relient le monde et avancent les possibilités de la rencontre. Ce n'est nullement un hasard que le soir de la conversation avec sa grand-mère, le narrateur entend « dans la nuit étoilée et froide les sifflements des locomotives ${ }^{27}$ » entre Paris et Doncières. C'est bien le sifflement des trains, « comme le chant d'un oiseau dans une forêt, relevant les distances ${ }^{28}$ ", qui est le premier son évoqué dans la Recherche. Ce chant de la locomotive resurgira comme un refrain d'espoir, un relais d'échos, ou plutôt d'écoutes, retransmis le long du roman fleuve, comme des satellites balayant leurs communications autour du globe.

\section{Albertine au téléphone}

Rien n'aiguise l'écoute autant que la jalousie. Une des plus belles scènes de téléphonie dans la Recherche survient dans Sodome et Gomorrhe, quand le narrateur, ayant quitté la soirée chez Mme de Guermantes, se hâte de rentrer chez lui pour accueillir Albertine qui doit lui rendre visite après avoir passé sa soirée, quant à elle, au théâtre. Le temps passe, Albertine le fait lanterner et, pour le narrateur perclus, l'écoute devient synonyme de souffrance :

Et je recommençai à écouter, à souffrir; quand nous attendons, de l'oreille qui recueille les bruits à l'esprit qui les dépouille et les analyse, et de l'esprit au cœur à 
qui il transmet ses résultats, le double trajet est si rapide que nous ne pouvons même pas percevoir sa durée, et qu'il semble que nous écoutions directement avec notre cœur ${ }^{29}$.

31 Mais il s'avère qu'écouter avec le cœur n'a rien de simple ; c'est un terrain piégé où il devient impossible de démêler les sources de manière objective. En fait, c'est plutôt, au contraire, une écoute indirecte, où le soupçon s'infiltre et déforme la parole de l'autre. Ces déformations, cette écoute de contre-espionnage est activée bien avant d'avoir l'autre à l'appareil. Tous les tropes que nous avons observés dans d'autres instances d'écoute sont réunis ici et l'attente silencieuse de l'appel d'Albertine fait fortement écho à la scène d'auscultation dans la chambre de Saint-Loup :

Il était trop tard, mais dans l'espoir que, soupant peut-être avec des amies, dans un café, elle aurait l'idée de me téléphoner, je tournai le commutateur rétablissant la communication dans ma chambre [...]. De peur de ne pas l'entendre, je ne bougeais pas. Mon immobilité était telle que, pour la première fois depuis des mois, je remarquai le tic-tac de la pendule. [...] arrivé au point culminant d'une ascension tourmentée dans les spirales de mon angoisse solitaire, du fond du Paris populeux et nocturne approché soudain de moi, à côté de ma bibliothèque, j'entendis tout à coup, mécanique et sublime, comme dans Tristan l'écharpe agitée ou le chalumeau du pâtre, le bruit de toupie du téléphone. Je m'élançai, c'était Albertine. «Je ne vous dérange pas en vous téléphonant à une pareille heure ? - Mais non ${ }^{30} .$. »

L'écoute du narrateur s'affine et se dilate, ciblant les sources, zoomant, si l'on peut dire, sur le tic-tac de la pendule (comme sur la montre de Saint-Loup) et se retirant pour un plan évocateur et un peu sournois de ce «Paris populeux et nocturne » qui l'entoure et dont pourrait surgir de toute part la menace d'une des amantes d'Albertine. Le jeu wagnérien offre une pléthore de références qui ajoutent à la scène ses nuances comiques et tragiques à la fois. Il y a bien sûr une chute du sublime au ridicule dans la comparaison des souffrances du narrateur avec celles du légendaire héros et son « écharpe agitée » ou avec le « chalumeau du pâtre » qui annonce l'arrivée du bateau d'Isolde dans le dernier acte de l'opéra. Mais on peut aussi songer à l'influence de l'écoute du théâtrophone qui semble faire irruption de nouveau dans le texte, fusionnant les sources radiophoniques.

33 L'effet est déroutant. L'analogie avec Tristan balaie les règles de style, accouplant Wagner à un coup de fil ; mais il n'y a rien de gratuit dans la provocation. En évoquant le Liebestod dans cette scène, Proust convoque, juste au-dessus du rire, la note tragique, le rappel de la mortalité, la disparition déjà annoncée ou présagée d'Albertine.

Mais avoir Albertine au téléphone ne résout rien non plus : il faut encore la convaincre de venir et Proust représente avec beaucoup de finesse le jeu et les manigances des amants qui tentent de se déjouer en obtenant ce qu'ils souhaitent sans donner l'apparence de trop le vouloir. C'est une véritable lutte verbale qui est menée et qui, comme elle passe entièrement par le fil téléphonique, est régie par les lois - ou disons les formes - de l'écoute. Merleau-Ponty décrit ce phénomène et en dresse un portrait magnifique qui fait directement écho à notre scène :

Quand j'écoute, il ne faut pas dire que j'ai la perception auditive des sons articulés, mais le discours se parle en moi; il m'interpelle et je retentis, il m'enveloppe et m'habite à tel point que je ne sais plus ce qui est de moi, ce qui est de lui. Dans les deux cas, je me projette en autrui, je l'introduis en moi, notre conversation ressemble à la lutte de deux athlètes aux deux bouts de l'unique corde ${ }^{31}$.

Les deux amants sont véritablement pris au piège des deux athlètes, pris en otage par un discours qui s'intercale entre eux au point de leur faire perdre les repères d'autrui. Proust fait éclore l'effet sous nos yeux et c'est une véritable phénoménologie du téléphone qu'il 
décrit, pour laquelle, que je sache, il n'existe aucun précédant dans la littérature moderne :

Mais ou était-elle? A ses paroles se mêlaient d'autres sons : la trompe d'un cycliste, la voix d'une femme qui chantait, une fanfare lointaine retentissaient aussi distinctement que la voix chère, comme pour me montrer que c'était bien Albertine dans son milieu actuel qui était près de moi en ce moment, comme une motte de terre avec laquelle on a emporté toutes les graminées qui l'entourent. Les mêmes bruits que j'entendais frappaient aussi son oreille et mettaient une entrave à son attention: détails de vérité, étrangers au sujet, inutiles en eux-mêmes, d'autant plus nécessaires à nous révéler l'évidence du miracle; traits sobres et charmants, descriptifs de quelque rue parisienne, traits perçants aussi et cruels d'une soirée inconnue qui, au sortir de Phèdre, avaient empêché Albertine de venir chez moi ${ }^{32}$.

Le téléphone synthétise les environs soniques, les livrant tissés ensemble, en une Gestalt qui ne peut être réduite à ces sources diverses. La voix d'Albertine n'est qu'une empreinte parmi les autres bourdonnements et rumeurs qui inondent le récepteur. Alors qu'il tente de communiquer, le narrateur est pris en écharpe par la texture contiguë du monde son collant -, cette étrangeté qui fait de chacun un ensemble qui ne peut être détaché de son contexte immédiat, qui emporte, comme la motte de terre, toutes ses "graminées » avec elle. Et il observe même cette curieuse mise en abîme de l'écoute, quand les mêmes brouillages externes entravent l'écoute de l'autre, qui tente de nous écouter !

Proust anticipe ici les paradoxes de la "haute fidélité », ce concept à la mode dans les nouvelles technologies de la reproduction du son. L'idée marchande, c'est la qualité véritable ou mimétique du son, c'est-à-dire la fidélité du son par rapport à sa source. Or, l'ironie, c'est que plus cette fidélité sonore s'aiguise, plus sa capacité à relever les « détails de vérité » s'accroît, plus augmente aussi, à l'inverse, l'évidence de l'infidélité d'Albertine, dévoilée par les indices révélateurs de ses alentours qu'elle ne peut tamiser.

Dans son étude sur Le Temps sensible, Julia Kristeva avait décrit deux horizons de l'expérience proustienne : «l'horizon extérieur des choses qui livrent leurs qualités à la clarté de notre conscience ; l'horizon intérieur de leurs ténèbres qui nous échappent ${ }^{33}$ ». Le téléphone, d'une manière unique, confond ces deux horizons. L'extérieur et l'intérieur s'interpénètrent. Le grain de la voix agglutiné à son environnement ne peut en être distingué : le contenant devient le contenu, comme le jeu des enfants plongeant leurs carafes dans les eaux de la Vivonne dans le célèbre passage de Du Côté de Chez Swann ${ }^{34}$.

Idéalement, le téléphone est un appareil à plier le temps et l'espace, une miniature ou un raccourci du but général de la Recherche, une sorte de looping au sein même du projet littéraire. Mais, on l'a bien vu, c'est un raccourci imparfait et inévitablement décevant, surtout lorsque il s'agit de contacter l'aimée. Car si le pli entre les deux amants les rapproche momentanément, il le fait tout en aiguisant l'angle qui les sépare. C'est un rapprochement illusoire qui place l'autre sur écoute et qui finit par transformer le dialogue en un jeu de contre-espionnage, un jeu où l'intelligence du cœur ne peut que tromper l'autre - et soi-même davantage.

\section{Conclusion : être appelé}

Nous n'avons en aucun cas réalisé une étude systématique de l'écoute dans la Recherche. Il y aurait une quantité d'autres scènes clefs qui n'ont pas été abordées ici. Pour n'en citer que quelques-unes, on pourrait mentionner le séjour du narrateur à Balbec avec sa grandmère, où celui-ci établit un système de communication à base de percussions et 
d'auscultation à travers la paroi, transformée en diaphragme, de leurs chambres contiguës. On pourrait s'attarder aussi sur la flagellation de Charlus mise "sur écoute " dans Le Temps retrouvé; ou sur le cri (le chant, la poésie) des vendeurs de légumes qui font le passe-muraille à travers la cellule où s'enferment le narrateur et Albertine dans $L a$ Prisonnière.

41 Il y a aussi des passages mineurs, mais également très curieux, comme le sifflement d'Albertine dans la douche entendu à travers le vitrage givré au tout début de $L a$ Prisonnière; ou le passage onirique de la voiture des amants dans la forêt de Chantepie dans Sodome et Gomorrhe, qui semble presque imiter l'effet d'une pièce de Debussy. On retrouverait partout les mêmes tropes et obsessions de l'écoute qu'on a tracés ici. On observerait aussi qu'à maintes reprises Proust revendique les libertés de l'invention romanesque qu'il applique à l'écoute, l'alignant avec sa conception de l'artiste moderne ${ }^{35}$. C'est d'ailleurs à seulement quelques pages de la fin du roman que le narrateur se livre à l'apologie suivante :

Mais enfin, je pourrais à la rigueur, dans la transcription plus exacte que je m'efforcerais de donner, ne pas changer la place des sons, m'abstenir de les détacher de leurs cause, à côté de laquelle l'intelligence les situe après coup, bien que faire chanter doucement la pluie au milieu de la chambre et tomber en déluge dans la cour l'ébullition de notre tisane ne dût pas être en somme plus déconcertant que ce qu'ont fait si souvent les peintres quand ils peignent ${ }^{36} .$.

Si Proust se tourne si explicitement vers ces transpositions de l'écoute, vers ces auscultations médiates, c'est-à-dire imaginatives, c'est qu'il est conscient de leur importance et qu'il souhaite ne pas les voir écartées comme des dérives stylistiques : elles ont un sens, un rôle spécifique à jouer dans la création d'une œuvre qui permet à ses lecteurs d'apprendre à écouter de nouveau, de s'entendre et d'être à l'écoute du monde d'une façon inhabituelle, comme un nouveau-né.

Proust voulait livrer ses lecteurs à eux-mêmes : «En réalité, chaque lecteur est, quand il lit, le propre lecteur de soi-même ${ }^{37}$. » C'est une manière de dire que chaque lecteur doit rester à l'écoute de son propre livre, du livre intérieur. Le corollaire, c'est qu'il faut croire à la parole - avoir foi dans le procédé littéraire. La lecture, si elle veut être autre chose qu'un passe-temps, doit permettre l'écoute de soi-même, une écoute qui, on l'a compris, passe aussi nécessairement par l'écoute de l'autre.

Or, aujourd'hui, l'échange de la parole et de l'écoute semble éclaté. Nos communications sont mises en réseau, fragmentées, surveillées en permanence par des entités parfois aussi banales que des agences de marketing, parfois aussi insidieuses que les organismes de sûreté gouvernementaux. Souvent, il est difficile de faire la différence. On pourrait parler d'un archipel de la parole, une sorte d'auréole qui entoure chacun de nous avec un archivage de nos dires et de nos écoutes en temps réel. La surécoute est devenu notre mode de vie. Ce sont autant de raisons pour examiner de près un phénomène dont Proust saisit l'élan initial et qui, d'une manière ou d'une autre, forme nos habitudes et nos mésententes quotidiennes. Chacun peut le constater: les scènes de téléphone dans la Recherche donnent une sensation d'actualité saisissante.

Lire la Recherche pour lire en nous-mêmes passe alors forcément par une interrogation de la place de l'écoute dans le roman. Il nous faut entendre notre propre écoute, surprendre la conversation que nous menons à chaque mot avec le texte: ce serait aussi une définition de l'enjeu de la critique littéraire et de son urgence dans un monde qui peine de plus en plus à s'entendre. 

filée de la parole à partir de la lecture du poème Un soir d'hiver de Georg Trakl. Pour Heidegger, la parole résonne comme la cloche du soir nous rappelant à la maison de l'Être. C'est une image qui évoque irrésistiblement le dernier - et sans doute le plus important - moment d'écoute dans la Recherche, celui où le narrateur se souvient du tintement de la petite sonnette qui annonçait le départ de Swann.

Appeler, interpeller, nommer : le texte de la Recherche place l'épiphanie dans la parole et dans l'écoute profonde : non celle des salons, mais celle qui nous ramène jusqu'au fond du jardin de la maison d'enfance, de la maison de Combray, cette maison dans le soir de l'Être, où, à l'étage, dans sa chambre, un petit garçon reste à l'écoute, attend le signe que sa mère viendra monter lui donner le baiser qui lui permettra de dormir. Heidegger: "Toute vraie écoute retient son propre dire. [...] Répondre, c'est être à l'écoute ${ }^{39}$.» Comprendre le texte proustien comme un acheminement vers l'écoute de l'Être, ce n'est peut être pas le plus mauvais résumé qu'on puisse faire d'un roman aussi vaste et complexe. L'importance de l'écoute est au cœur du roman, elle l'irrigue et fait le relais, comme le sifflement des trains dans la campagne, entre les tomes, traversant sans délai les épaisseurs du Temps. Il ne faut pas le croire sur parole. Écoutons plutôt :

[...] ce bruit des pas de mes parents reconduisant M. Swann, ce tintement rebondissant, ferrugineux, intarissable, criard et frais de la petite sonnette qui m'annonçait qu'enfin M. Swann était parti et que maman allait monter, je les entendis encore, je les entendis eux-mêmes, eux situés pourtant si loin dans le passé. [...] C'est donc que ce tintement y était toujours, et aussi, entre lui et l'instant présent, tout ce passé indéfiniment déroulé que je ne savais que je portais. Quand elle avait tinté, j'existais déjà, et depuis, pour que j'entendisse encore ce tintement, il fallait qu'il n'y eût pas de discontinuité, que je n'eusse pas un instant cessé d'exister, de penser, d'avoir conscience de $\mathrm{moi}^{40}{ }^{40}$.

\section{NOTES}

1. SZENDY, Peter, Sur écoute. Esthétique de l'espionnage, Paris, Éditions de Minuit, 2007, p. 26. Ma lecture de Proust puise son inspiration dans le séminaire exceptionnel de Peter Szendy sur la théorie de la ponctuation à l'université de Princeton au printemps 2012. Qu'il en soit ici remercié. 2. RICHARD, Jean-Pierre Proust et le monde sensible, Paris, Éditions du Seuil, 1974. Ce livre traite pourtant minutieusement de tous les autres sens, par exemple le dur et le mou, l'aéré, le velouté, le soyeux, le fourré, le tacheté, le feuillu, l'odorant, l'écumeux. Il comprend, par contre, un chapitre sur la "petite phrase" de Vinteuil, mais l'analyse se limite à cette musique sans s'attacher aux autres ambiances sonores du roman.

3. ALBARET, Céleste, Monsieur Proust, souvenirs recueillis par Georges Belmont, Paris, Robert Laffont, 1973.

4. Philippe Sollers évoque une « écriture sismique » dans le manuscrit proustien (Éloge de l'infini, Paris, Gallimard, 2003).

5. Cf. SZENDY, Peter, À coups de points. La ponctuation comme expérience, Paris, Éditions de Minuit, 2013, notamment le chapitre intitulé « Les pointillés de l'auscultation ». 
6. LAENNEC, René-Théophile-Hyacinthe, De l'auscultation médiate ou Traite du diagnostic des maladies des poumons et du cour, Paris, Brosson et Chaudé, 1819.

7. Foucault en a fait l'objet de sa critique dans Naissance de la Clinique (Presses universitaires de France, 1963), où il place Laennec parmi les médecins et scientifiques de la fin du XVIII ${ }^{\mathrm{e}}$ siècle qui dépècent le corps humain sous l'œil « objectif » de la médecine.

8. PROUST, Marcel, À la recherche du temps perdu, Paris, Gallimard, «La Pléiade », 1954 : Le Côté de Guermantes, II, p. 73-4.

9. Ibid., p. 73-4.

10. Ibid., p. 74.

11. Ibid., p. 75 .

12. Ibid., p. 76.

13. Cf. Le temps retrouvé, III, p. 919-920: "N'est-ce pas à une sensation du genre de celle de la madeleine qu'est suspendue la plus belle partie des Mémoires d'Outre-Tombe: "Hier au soir je me promenais seul... je fus tiré de mes réflexions par le gazouillement d'une grive perchée sur la plus haute branche d'un bouleau." » Et plus loin : " une sensation du même genre que le goût de la madeleine et le "gazouillement de la grive" ".

14. Le Côté de Guermantes, II, p. 76.

15. Ibid., p. 76.

16. PERRON, «Le théâtrophone », dans Le Magasin pittoresque, 15 juin 1892, p. 183-184.

17. Le 21 février 1911, Proust écrit à son ami Reynaldo Hahn : "J'ai entendu hier au théâtrophone un acte des Maîtres Chanteurs [...] et ce soir... tout Pelléas » (Lettres à Reynaldo Hahn, présentées, datées et annotées par Philip Kolb, Paris, Gallimard, 1956, p. 199). Kolb observe que l'abonnement de Proust paraît lié à une nouvelle campagne promotionnelle du théâtrophone cette année-là et cite une annonce parue dans le Tout Paris de 1911. Sur l'influence de l'opéra dans l'œuvre proustien, notamment à travers le théâtrophone, voir NEWARK, Cormac et WASSENAAR, Ingrid, «Proust and Music: The Anxiety of Competence », Cambridge Opera Journal, vol. $9 \mathrm{n}^{\circ} 2$, juillet 1997, p. 163-183.

18. Le Côté de Guermantes, II, p. 77.

19. DELEUZE, Gilles, Marcel Proust et les signes, Paris, Presses universitaires de France, 1964, p. 39.

20. SZENDY, Peter, À coups de points, Paris, Éditions de Minuit, 2013, p. 70.

21. Cf. PAINTER, George, Proust. The Early Years, Boston, Little Brown, 1954, p. 242-243 : «On October 20, the day after his departure, Mme Proust had crossed the Boulevard to Cerisier's bakery at Number 8 to telephone her son. "But Cerisier's subscription didn't include calls outside Paris, and despite all my efforts to the Ladies of the Telephone to pay extra, they banished me to the public cabin." [...] "When her poor voice reached me," [Proust] wrote to Antoine Bibesco six years later, "it was broken and bruised, for ever changed from the voice I had known, full of cracks and crevices; and it was only when I reconstituted in the telephone receiver those shattered and bleeding fragments of words, that I had for the first time the horrible feeling of all that was broken inside her." "

22. PROUST, Marcel, Jean Santeuil, Paris, Gallimard, 2001, p. 417-418.

23. Le Côté de Guermantes, II, p. 133.

24. Ibid., p. 134.

25. Ibid., p. 135.

26. Ibid., p. 135.

27. Ibid., p. 137.

28. Cf. Du Côté de chez Swann, I, p. 3: "J'entendais le sifflement des trains qui, plus ou moins éloigné, comme le chant d'un oiseau dans un forêt, relevant les distances, me décrivait l'étendue de la campagne déserte où le voyageur se hâte vers la station prochaine ».

29. Sodome et Gomorrhe, II, p. 731.

30. Ibid., p. 730-731.

31. MERLEAU-PONTY, Maurice, La prose du monde, Paris, Gallimard, 1969, p. 28-29. 
32. Sodome et Gomorrhe, II, p. 732.

33. KRISTEVA, Julia, Le Temps sensible, Paris, Gallimard, 1994, p. 332.

34. Cf. Du Côté de chez Swann, I, p. 166: «Je m'amusais à regarder les carafes que les gamins mettaient dans la Vivonne pour prendre les petits poissons, et qui, remplies par la rivière, où elles sont à leur tour encloses, à la fois "contenant" aux flancs transparents comme une eau durcie, et "contenu" plongé dans un plus grand contenant de cristal liquide ».

35. Cf. La Prisonnière, III, p. 606-607: «Pendant qu'Albertine allait ôter ses affaires, et pour aviser au plus vite, je me saisis du récepteur du téléphone, j'invoquai les Divinités implacables, mais ne fis qu'exciter leur fureur qui se traduisit par ces mots : "Pas libre." Andrée était en train en effet de causer avec quelqu'un. En attendant qu'elle eût achevé sa communication, je me demandais comment, puisque tant de peintres cherchent à renouveler les portraits féminins du XVIII ${ }^{\mathrm{e}}$ siècle où l'ingénieuse mise en scène est un prétexte aux expressions de l'attente, de la bouderie, de l'intérêt, de la rêverie, comment aucun de nos modernes Boucher ou Fragonard, ne peignit, au lieu de La Lettre, du Clavecin, etc., cette scène qui pourrait s'appeler: Devant le téléphone, et où naîtrait spontanément sur les lèvres de l'écouteuse un sourire d'autant plus vrai qu'il sait n'être pas vu. »

36. Le Temps retrouvé, III, p. 1045.

37. Ibid., III, p. 911.

38. HEIDEGGER, Martin, «La parole », dans Acheminement vers la parole, traduction française de François Fédier, Paris, Gallimard, 1976. C'est de la première strophe du poème de Trakl qu'il est question : Quand il neige à la fenêtre / Que longuement sonne la cloche du soir, / Pour beaucoup la table est mise / Et la maison est bien pourvue.

39. Ibid., p. 36.

40. Le Temps retrouvé, III, p. 1046-1047.

\section{RÉSUMÉS}

$\mathrm{Au}$ cœur de la Recherche du temps perdu se trouve une réflexion sur les nouvelles technologies téléphoniques qui révolutionnent la phénoménologie de l'écoute et de la communication au début $\mathrm{du} \mathrm{XX}^{\mathrm{e}}$ siècle. L'expérience de la parole à distance, le relais ou la reproduction des sources sonores relayées et, surtout, l'écoute médiatisée ou l'« auscultation » dominent l'imaginaire de l'auralité dans l'œuvre proustien. L'article se propose d'appliquer les concepts de « surécoute » et d'«auscultation médiate» - deux stratégies de lectures développées par le philosophe et musicologue Peter Szendy - pour renouveler l'analyse du texte et réévaluer l'écoute en tant que thématique majeure du roman.

\section{INDEX}

Mots-clés : Proust, écoute, auscultation, téléphone, parole, surécoute, médiation, son 


\section{AUTEUR}

\section{JESSE DYLAN MCCARTHY}

Jesse McCarthy achève actuellement son doctorat dans le département d'anglais à l'université de Princeton. Sa thèse - Out in the Cold. Strategies of Black Writing in the Early Cold War, 1945-1972 retrace les tensions identitaires au sein des mouvements littéraires afro-américains à l'époque de la guerre froide. A Princeton, il a été le fondateur du projet de numérisation « Mapping Expatriate Paris », qui permettra de rendre disponibles en ligne les archives de Sylvia Beach et de sa célèbre librairie, Shakespeare and Company. Ses recherches se portent sur l'identité, la technologie et l'esthétique dans les mouvements littéraires au XX $\mathrm{X}^{\mathrm{e}}$ siècle. Ayant fait ses études secondaires en France, il s'intéresse aussi à la traduction et à la critique littéraire françaises et francophones. 